\section{Response to Juang et al.}

To the Editor

We appreciate the interesting response by Juang et al. ${ }^{1}$ to our article. ${ }^{2}$ The authors provide a completely different point of view and argue that IVS4+919 G>A is not a diseasecausing variant per se, but just a benign marker for other pathogenic variants that cause hypertrophic cardiomyopathy (HCM). However, to us the mounting weight of evidence points toward a direct involvement of the IVS4+919 G>A variant in the pathogenesis of this form of Fabry disease. First, the variant is in the galactosidase A gene and results in the production of a defective enzyme. Second, patients with the IVS4+919 G>A variant have an accumulation of glycosphingolipid substrate (Gb3) in their hearts at both early and late stages, ${ }^{2}$ including the three patients reported by Juang et al. Third, the association of IVS4+919 G>A variant with the later-onset phenotype of Fabry disease manifested as HCM has been shown in multiple publications, ${ }^{3-7}$ and patients carrying the IVS4+919 G>A variant with cardiac symptoms are continuously being identified worldwide. Juang et al. argued that the reported three patients only had mild elevation of plasma lysoGb3 $(3.35-4.33 \mathrm{ng} / \mathrm{mL}, N<0.8)$ and suggested that Fabry disease might not be the major pathological factor for their cardiomyopathy. Nevertheless, we consider this a premature conclusion because the classical Fabry disease-associated pathologic changes, such as inclusion bodies, do exist in the endocardial biopsies of these patients. This argument truly reflects the difficulties in diagnosis due to the nonspecific nature of the inclusion bodies, yet highlights the importance of using Gb3-specific detection methods as we suggested. ${ }^{2}$

Most likely, the disagreement whether or not IVS4+919 $\mathrm{G}>\mathrm{A}$ is a major pathogenic variant stems from the different patient cohorts used in the different studies. For example, screening efforts focused on hypertrophic cardiomyopathy patients found that about $1 \%$ of those screened had GLA variants. ${ }^{8-10}$ On the other hand, in a recent publication, Chiang et al. ${ }^{11}$ made their argument based on screening results in patients with a much broader spectrum of heart conditions including heart failure, ventricular tachycardia, atrial fibrillation, and coronary artery disease, instead of focusing on left ventricular hypertrophy or HCM, leading to the relative paucity of clinically affected patients with the IVS4 $+919 \mathrm{G}>\mathrm{A}$ variant found in their study.

In the present communication, Juang et al. discussed the possibility that additional genetic modifiers in association with the IVS4+919 G>A variant may have impact on the severity of the clinical symptoms. Their exome sequencing
(ES), although limited to only three patients, revealed several gene variations related to cardiomyopathy and arrhythmia with unknown significance. This method is a great addition to the current understanding of the IVS4+919 G>A pathogenesis and underlines the urgent need for more extensive and indepth studies with sufficient number of samples analyzed.

In our opinion, there could exist many modifying factors that affect Fabry disease, especially late-onset Fabry disease. Our unpublished results suggest that not only genetic factors but also acquired factors, such as smoking, obesity, diabetes, hypertension, or hypercholesterolemia, can contribute to the severity of IVS4+919 G>A cardiomyopathy. We believe that ES or genome sequencing (GS) will be important methods to approach the question about genetic modifiers of Fabry disease, and detailed analyses from many more ES/GS studies would be invaluable to further improve our knowledge and treatment of Fabry disease.

\section{DISCLOSURE}

The authors declare no conflicts of interest.

Publisher's note: Springer Nature remains neutral with regard to jurisdictional claims in published maps and institutional affiliations.

\section{Chia-Lin Hsu, PhD (1) ${ }^{1}$, Ivan L. Dzhagalov, $M D, P h D^{1}$ and Dau-Ming Niu, MD, $P h D^{2,3,4}$}

${ }^{1}$ Institute of Microbiology and Immunology, National Yang-Ming University, Taipei, Taiwan; ${ }^{2}$ Institute of Clinical Medicine, National Yang-Ming University, Taipei, Taiwan; ${ }^{3}$ Department of Pediatrics, Taipei Veterans General Hospital, Taipei, Taiwan; ${ }^{4}$ Department of Medical Research, Taipei Veterans General Hospital, Taipei, Taiwan. Correspondence: Chia-Lin Hsu (Chialin.hsu@ym.edu.tw) or Dau-Ming Niu (dmniu1111@yahoo.com.tw)

\section{REFERENCES}

1. Juang C-M, Don S-J, Hwu W-L et al. Fabry disease cardiac variants IVS4 $+919 \mathrm{G}>\mathrm{A}$ is associated with multiple cardiac gene mutations in patients with severe cardiomyopathy and fatal arrhythmia. Genet Med. 2019.

2. Hsu M-J, Chang F-P, Lu Y-H, et al. Identification of lysosomal and extralysosomal globotriaosylceramide (Gb3) accumulations before the occurrence of typical pathological changes in the endomyocardial biopsies of Fabry disease patients. Genet Med. 2018 Jun 6; https://doi. org/10.1038/s41436-018-0010-z. [Epub ahead of print].

3. Nakao S, Takenaka T, Maeda M, et al. An atypical variant of Fabry's disease in men with left ventricular hypertrophy. N Engl J Med. 1995;333:288-293.

4. Spada M, Pagliardini S, Yasuda $\mathrm{M}$, et al. High incidence of later-onset fabry disease revealed by newborn screening. Am J Hum Genet. 2006:79:31-40

5. Inoue T, Hattori K, Ihara K, Ishii A, Nakamura K, Hirose S. Newborn screening for Fabry disease in Japan: prevalence and genotypes of Fabry disease in a pilot study. J Hum Genet. 2013:58:548-552.

6. Hsu T-R, Sung S-H, Chang F-P, et al. Endomyocardial biopsies in patients with left ventricular hypertrophy and a common Chinese later-onset fabry mutation (IVS4+919G>A). Orphanet J Rare Dis. 2014;9:96.

7. Hsu TR, Hung SC, Chang FP, et al. Later onset Fabry disease, cardiac damage progress in silence: experience with a highly prevalent mutation. J Am Coll Cardiol. 2016;68:2554-2563. 
8. Sachdev B, Takenaka T, Teraguchi H, et al. Prevalence of Anderson-Fabry disease in male patients with late onset hypertrophic cardiomyopathy. Circulation. 2002:105:1407-1411.

9. Monserrat L, Gimeno-Blanes JR, Marín F, et al. Prevalence of fabry disease in a cohort of 508 unrelated patients with hypertrophic cardiomyopathy. J Am Coll Cardiol. 2007:50:2399-2403.

10. Elliott $P$, Baker R, Pasquale $F$, et al. Prevalence of Anderson-Fabry disease in patients with hypertrophic cardiomyopathy: the European Anderson-Fabry Disease Survey. Heart. 2011;97:1957.
11. Chiang H-L, Wang NH-H, Song I-W, et al. Genetic epidemiological study doesn't support GLA IVS4+919G >A variant is a significant mutation in Fabry disease. Mol Genet Metab. 2017;121:22-27.

Advance online publication 22 January 2019. doi:10.1038/s41436-019-0437-x 